\title{
Saúde e lazer entre idosos diabéticos rurais com e sem indicativo de depressão
}

\section{Health and leisure among elderly rural diabetics with and without indications of depression Salud y ocio entre los mayores diabéticos rurales con y sin indicador de depresión}

\author{
Darlene Mara dos Santos Tavares'; Tamires Gomes dos Santos ${ }^{\text {II }}$; Flavia Aparecida Dias ${ }^{I I I}$; \\ Alisson Fernandes Bolina ${ }^{I V}$; Pollyana Cristina dos Santos Ferreira ${ }^{V}$
}

\begin{abstract}
RESUMO: O objetivo deste estudo foi comparar as condições de saúde e lazer dos idosos diabéticos com e sem indicativo de depressão. Foram entrevistados 104 idosos diabéticos da zona rural de Uberaba-Minas Gerais, em 2011, utilizando-se os instrumentos Escala de Depressão Geriátrica Abreviada e semiestruturado, baseado no Olders Americans Resoucers and Services. Realizou-se análise descritiva e teste quiquadrado $(p<0,05)$. A maioria, em ambos os grupos, era do sexo feminino, faixa etária entre 60-70 anos, moravam com o esposo ou companheiro, de 4-8 anos de estudo e renda de até um salário mínimo. Os idosos diabéticos com indicativo de depressão apresentaram, proporcionalmente, pior autopercepção da saúde, mais complicações relacionadas ao diabetes, maior uso de medicamentos combinados e insulina. Evidencia-se a necessidade de ações de saúde direcionadas ao monitoramento dos idosos diabéticos com indicativo de depressão visando estabelecimento de diagnóstico e acompanhamento para minimizar o impacto da doença no seu cotidiano.

Palavras-Chave: Idoso; diabetes mellitus; população rural; depressão.
\end{abstract}

\begin{abstract}
This study compared the health and recreation of elderly diabetics with and without indications of depression. We interviewed 104 elderly diabetics in rural areas of Uberaba, Minas Gerais, Brazil, in 2011 using as instruments the Geriatric Depression Scale Short and a semistructured based on Older Americans Resources and Services. Descriptive analysis and chisquare test $(p<0.05)$ were performed. Most participants in both groups were female, aged 60-70 years, lived with a husband or partner, had 4-8 years' schooling, and income of one minimum wage. The elderly diabetics with indications of depression had proportionally worse self-perceived health, more diabetes-related complications, and greater use of drug combinations and insulin. This confirms the need for health measures to monitor elderly diabetics with indications of depression, with a view to establishing diagnosis and following up to minimize the impact of the disease on their daily lives.

Keywords: Older adults; diabetes mellitus; rural population; depression.
\end{abstract}

RESUMEN: El objetivo de este estudio fue comparar las condiciones de salud y de ocio de personas mayores diabéticas con y sin indicación de la depresión. Se entrevistaron 104 pacientes mayores diabéticos de la zona rural de Uberaba - Minas Gerais, Brasil, en 2011, utilizando los instrumentos Escala de Depresión Geriátrica Abreviada, semiestructurados sobre la base de Olders Americans Resoucers and Services. Se realizó el análisis descriptivo y prueba de chi cuadrado ( $<<0,05)$. La mayoría, en ambos grupos, era del sexo femenino, entre 60 y 70 años, vivía con su esposo o compañero, tenía 4 a 8 años de escolaridad e ingresos de hasta un salario mínimo. Los mayores diabéticos con indicios de depresión presentaron, proporcionalmente, peor autopercepción de la salud, más complicaciones relacionadas con la diabetes, mayor uso de medicamentos combinados e insulina. Esto confirma la necesidad de acciones de salud dirigidas al monitoreo de los mayores diabéticos con indicios de depresión con vistas a establecer el diagnóstico y el seguimiento para minimizar el impacto de la enfermedad en sus vidas diarias.

Palabras Clave: Mayores; diabetes mellitus; población rural; depresión.

\section{INTRODUÇÃO}

Segundo o Instituto Brasileiro de Geografia e Estatística (IBGE), no ano de 1999, os idosos representavam, aproximadamente, $9,1 \%$ da população brasileira e, em $2009,11,3 \%{ }^{1}$.
Essa elevação na proporção de idosos no país apresenta relação com o processo de transição epidemiológica, caracterizado pela alteração do perfil de morbidade e de mortalidade, a qual apresentou uma

'Doutor em Enfermagem. Professor Associado do Departamento de Enfermagem em Educação e Saúde Comunitária do Curso de Graduação em Enfermagem da Universidade Federal do Triângulo Mineiro. Uberaba, Minas Gerais, Brasil. E-mail: darlenetavares@enfermagem.uftm.edu.br.

IIAcadêmica do Curso de Graduação em Enfermagem da Universidade Federal do Triângulo Mineiro. Bolsista de Iniciação Científica financiada pelo Conselho Nacional de Desenvolvimento Científico e Tecnológico. Uberaba, Minas Gerais, Brasil. E-mail: tamiresgomes_santos@hotmail.com.

IIIMestre em Atenção à Saúde. Professor Substituto do Departamento de Enfermagem em Educação e Saúde Comunitária do Curso de Graduação em Enfermagem da Universidade Federal do Triângulo Mineiro. Uberaba-Minas Gerais, Brasil. E-mail: flaviadias_ura@yahoo.com.br.

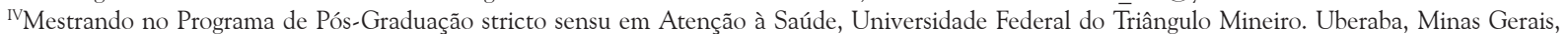
Brasil. E-mail: alissonbolina@yahoo.com.br.

vMestre em Atenção à Saúde. Professor Substituto do Departamento de Medicina Social da Universidade Federal do Triângulo Mineiro. Uberaba, Minas Gerais, Brasil. E-mail: pollycris21@bol.com.br. 
diminuição progressiva das mortes por doenças infectocontagiosas e aumento das morbidades crônicas². Entre essas, destaca-se o Diabetes Mellitus (DM).

De acordo com a Pesquisa Nacional por Amostra de Domicílios, em 2008, 16,1\% dos idosos brasileiros apresentavam $\mathrm{DM}^{1}$. Destaca-se que o Brasil é um país primariamente agrário e muitos de seus municípios são enquadrados como rurais. Apesar disso, as pesquisas sobre o envelhecimento têm ocorrido, principalmente, em áreas urbanas, gerando uma lacuna sobre as reais necessidades da população rural $^{3}$. Assim, este estudo fará um recorte para a população idosa rural com DM, visando contribuir para a política de atenção à saúde desses idosos.

Desse modo, os objetivos deste estudo foram: descrever as características sociodemográficas e econômicas de idosos diabéticos com e sem indicativo de depressão e comparar as condições de saúde e lazer entre idosos diabéticos com e sem indicativo de depressão, residentes no espaço rural.

\section{REVISÃO DE LITERATURA}

Inquérito na Suécia observou predomínio do DM entre os idosos da zona rural $(16,1 \%)$ em relação aos que residiam no espaço urbano $(6,3 \%)^{4}$. No Brasil, investigação conduzida no interior de Minas Gerais observou que os idosos residentes em áreas rurais apresentavam menores chances de terem a doença ${ }^{5}$.

O DM faz parte de um grupo de doenças metabólicas, caracterizada pelo excesso de glicemia, resultante de um defeito na secreção e/ou ação da insulina ${ }^{6}$. Além disso, evidencia-se que esta morbidade pode favorecer sentimentos de impotência bem como a ocorrência de sintomas depressivos?

Uma possível explicação para o desenvolvimento de depressão em pessoas com DM está relacionada à atividade do eixo adenohipofisário-adrenocortical, ocasionando descontrole metabólico. Assim, ocorre hiperglicemia devido à estimulação da gliconeogênese hepática, degradação de proteínas, elevação do número de glicocorticoides em tecido adiposo e inibição dos receptores de glicose nos tecidos adiposo e muscular. Além disso, ocorre o bloqueio dos receptores serotoninérgicos 5HT, predispondo ao desenvolvimento da depressão ${ }^{8}$.

Apesar disto, não há consenso na literatura científica da relação do DM e depressão. Estudo realizado com idosos na zona rural da Índia verificou associação entre a depressão e o DM ${ }^{9}$. Porém, outra pesquisa obteve que idosos com DM não são mais afetados pela depressão em relação àqueles que apresentam outras condições crônicas ${ }^{10}$.

Além disso, estudos desenvolvidos no Brasil verificaram que os sintomas depressivos estão associados ao analfabetismo, sexo feminino, idade avançada, presença de comorbidades, baixa escolaridade, uso de medicamentos, pouca atividade física e menor autocuidado em pessoas idosas com $\mathrm{DM}^{11,12}$.

Ressalta-se que os indivíduos com DM têm seu tratamento voltado às mudanças na alimentação, ao uso de medicamentos e à prática de atividade física. Assim, no decorrer de seu tratamento, os sujeitos podem vivenciar sentimentos que dificultam a aceitação da doença; consequentemente, na adoção de hábitos saudáveis ${ }^{7}$. Infere-se que estes fatores, associados ao indicativo de depressão, podem impactar negativamente sobre suas condições de saúde.

\section{Metodologia}

O presente estudo faz parte do projeto Saúde e qualidade de vida da população idosa da zona rural de Uberaba desenvolvido pelo Grupo de Pesquisa em Saúde Coletiva da Universidade Federal do Triângulo Mineiro.

Trata-se de um inquérito domiciliar, transversal e observacional, desenvolvido na zona rural do município de Uberaba-MG. Esta localidade apresenta cobertura de 100\% das áreas de abrangências, realizada por quatro equipes da estratégia saúde da família (ESF).

A população foi composta por 1.297 idosos que residem na zona rural do município de Uberaba-MG. As ESF disponibilizaram uma lista contendo o nome e o endereço dos idosos cadastrados, mediante autorização da Secretaria Municipal de Saúde.

Os critérios de inclusão foram: ter 60 anos ou mais de idade, residir na zona rural do município de Uberaba-MG, não ter declínio cognitivo, autorreferir DM e aceitar participar do estudo.

Do total de 1.297, foram excluídos 447 idosos por: mudança de endereço (117), declínio cognitivo (105), recusa (75), não encontrados após três tentativas do entrevistador (57), óbito (11), hospitalização (3) e outros motivos (57). Assim, foram entrevistados 850 idosos; destes, 104 atenderam aos critérios de inclusão da presente pesquisa. Considerando o escore obtido na Escala de Depressão Geriátrica Abreviada, constituíram-se dois grupos: idosos com DM e indicativo de depressão $(n=32)$ e idosos com DM e sem indicativo de depressão $(n=72)$.

As entrevistas foram realizadas no domicílio do idoso, por 14 entrevistadores, devidamente treinados. Contou-se com a colaboração dos agentes comunitários de saúde para a localização da residência. Os dados foram coletados no período de junho de 2010 a março de 2011.

As entrevistas foram revisadas por supervisores de campos, os quais verificavam questões incompletas e inconsistência das respostas. Quando houve necessidade, a entrevista foi devolvida ao entrevistador para realização do preenchimento adequado. 
O declínio cognitivo foi avaliado pelo Mini Exame do Estado Mental (MEEM), traduzido e validado no Brasil. O ponto de corte foi: 13 para analfabetos, 18 para 1 a 11 anos de estudo e 26 para acima de 11 anos $^{13}$.

Para avaliar o indicativo de depressão, foi utilizada a Escala de Depressão Geriátrica Abreviada (EDG-15), versão adaptada no Brasil ${ }^{14}$.

Para caracterização dos dados sociodemográficos, econômicos, de saúde e lazer, foi aplicado parte do questionário Olders Americans Resoucers and Services (OARS), adaptado à realidade brasileira ${ }^{15}$.

Investigaram-se as variáveis sociodemográficas e econômicas (sexo; faixa etária; estado conjugal; escolaridade; renda individual mensal, em salários mínimos; arranjo de moradia; razão de aposentadoria); saúde (autopercepção de saúde; comparação da saúde atual com a de 12 meses atrás; comparação com a saúde de outras pessoas; consulta mensal; local da consulta; cadastro no Hiperdia; tempo de diagnóstico de DM; complicações; tipo de complicações; uso de medicação; tipo de medicação); lazer (realização de lazer; passatempo; satisfação nas atividades de lazer).

Foi construído um banco de dados eletrônico, no programa Excel ${ }^{\circledR}$, e os dados coletados foram processados em dupla entrada por dois digitadores. O banco de dados foi transportado para o software Statiscal Package for the Social Sciences (SPSS), versão 17.0, para realização da análise.

Os dados foram submetidos à análise descritiva por meio das frequências absolutas e percentuais. Para comparação dos grupos, aplicou-se o teste quiquadrado para as variáveis categóricas $(p<0,05)$.

O presente trabalho foi aprovado pelo Comitê de Ética em Pesquisa com Seres Humanos da Universidade Federal do Triângulo Mineiro, sob protocolo $\mathrm{n}^{\circ} 1477$. Após a assinatura do Termo de Consentimento Livre e Esclarecido, conduziu-se a entrevista com o idoso.

\section{Resultados e Discussão}

As características sociodemográficas e econômicas da população estudada são apresentadas na Tabela 1.

Em ambos os grupos, a maioria era do sexo feminino com maior percentual entre os idosos diabéticos com indicativo de depressão em relação aos sem, conforme mostra a Tabela 1. Estes dados corroboram com investigação realizada no Sul do Brasil, a qual verificou que $83,3 \%$ dos idosos diabéticos com depres-

TABELA 1: Distribuição de frequência das variáveis sociodemográficas e econômicas dos idosos da zona rural com e sem indicativo de depressão. Uberaba, Minas Gerais, 2012.

\begin{tabular}{|c|c|c|c|c|c|}
\hline \multirow{2}{*}{ Variáveis } & & \multicolumn{2}{|c|}{ Com } & \multicolumn{2}{|c|}{ Sem } \\
\hline & & $f$ & $\%$ & f & $\%$ \\
\hline \multirow[t]{2}{*}{ Sexo } & Feminino & 26 & 81,2 & 42 & 58,3 \\
\hline & Masculino & 6 & 18,8 & 30 & 41,7 \\
\hline \multirow[t]{3}{*}{ Faixa etária (em anos) } & $60-70$ & 19 & 59,4 & 45 & 62,5 \\
\hline & $70-80$ & 8 & 25,0 & 22 & 30,6 \\
\hline & 80 e mais & 5 & 15,6 & 5 & 6,9 \\
\hline \multirow[t]{4}{*}{ Estado conjugal } & Nunca se casou ou morou com companheiro(a) & 2 & 6,2 & 7 & 9,7 \\
\hline & Mora com o esposo(a) ou companheiro(a) & 18 & 56,2 & 49 & 68,1 \\
\hline & Viúvo & 12 & 37,5 & 14 & 19,4 \\
\hline & Separado, desquitado, divorciado & - & - & 2 & 2,8 \\
\hline \multirow[t]{6}{*}{ Arranjo de moradia } & Só & 5 & 15,6 & 9 & 12,5 \\
\hline & Somente com o cônjuge & 12 & 37,5 & 35 & 48,6 \\
\hline & Com outros de sua geração (com/sem o cônjuge) & 3 & 9,4 & 7 & 9,7 \\
\hline & Com filhos (com ou sem o cônjuge) & 10 & 31,2 & 16 & 22,2 \\
\hline & Com netos (com ou sem o cônjuge) & 1 & 3,1 & 2 & 2,8 \\
\hline & Outros arranjos & 1 & 3,1 & 3 & 4,2 \\
\hline \multirow{6}{*}{$\begin{array}{l}\text { Renda Individual (em salários } \\
\text { mínimos) }{ }^{(*)}\end{array}$} & Sem renda & 3 & 9,4 & 11 & 15,3 \\
\hline & $<1$ & 1 & 3,1 & 3 & 4,2 \\
\hline & 1 & 15 & 46,9 & 28 & 38,9 \\
\hline & $1-3$ & 12 & 37,5 & 21 & 29,2 \\
\hline & $3-5$ & 1 & 3,1 & 7 & 9,7 \\
\hline & $>5$ & - & - & 2 & 2,8 \\
\hline \multirow[t]{4}{*}{ Razão de Aposentadoria } & Tempo de serviço & 6 & 18,8 & 22 & 30,6 \\
\hline & Idade & 8 & 25,0 & 24 & 33,3 \\
\hline & Problema de saúde & 7 & 21,9 & 8 & 11,1 \\
\hline & Não se aposentou & 11 & 34,4 & 18 & 25,0 \\
\hline
\end{tabular}

(*) Salário mínimo no período da coleta: R\$545,00, DIEESE, 2012. 
são e $65,2 \%$ daqueles sem depressão eram mulheres ${ }^{16}$. Tal fato pode estar relacionado à maior preocupação das mulheres com sua saúde e, com isso, serem mais frequentes nos serviços de saúde, gerando uma maior possibilidade de realização do diagnóstico da doença e, consequentemente, tratamento ${ }^{17}$.

Prevaleceram, tanto para os idosos diabéticos com e sem indicativo de depressão, a faixa etária de 60-70 anos, segundo a Tabela 1. Destaca-se que $5(15,6 \%)$ idosos diabéticos com indicativo de depressão tinham 80 anos e mais, de acordo com a Tabela 1. Esse resultado é condizente com investigação realizada no sul do Brasil, em que 58,7\% dos idosos com DM e depressão e 60,2\% dos idosos apenas com DM encontravam-se entre 60-70 anos ${ }^{16}$. Evidencia-se que a maioria desses indivíduos são idosos jovens, o que denota a necessidade de criar estratégias que auxiliem no enfrentamento dessa doença ao longo da vida. $\mathrm{O}$ rastreamento da depressão nos idosos diabéticos deve ser realizado periodicamente, sendo a Escala Geriátrica de Depressão um instrumento expressivo na detecção de indicativo de depressão em idosos ${ }^{17}$.

No entanto, destaca-se que o elevado percentual de idosos diabéticos com indicativo de depressão em idades mais avançadas pode ser elucidado pelo excesso de perdas física, mental e social que ocorrem ao longo dos anos, favorecendo o aparecimento de sintomas depressivos nesses indivíduos ${ }^{11}$.

Em ambos os grupos, a maioria possuía esposo(a)/ companheiro(a) e residia somente com o cônjuge. Entretanto, $12(37,5 \%)$ idosos diabéticos com indicativo de depressão eram viúvos, conforme mostra a Tabela 1. Esse resultado é convergente ao da pesquisa conduzida na zona rural dos Estados Unidos, que verificou percentual de idosos com DM casados em ambos os grupos de indivíduos com $(39,1 \%)$ e sem sintomas depressivos $(52,1 \%)^{18}$. Este fato pode estar relacionado à migração dos filhos para a zona urbana na busca de oportunidades de trabalho ou estudo ${ }^{19}$.

No entanto, a alta prevalência de viuvez entre idosos diabéticos com indicativo de depressão pode ser decorrente dos sentimentos vivenciados por esses indivíduos durante a fase de luto. A perda recente de parentes, especialmente do companheiro, contribui para sintomatologia depressiva ${ }^{11}$. Adicionalmente, algumas situações desfavoráveis do próprio processo de envelhecimento podem também favorecer o aparecimento desse sintoma, como a diminuição da saúde, da capacidade cognitiva, dos recursos financeiros e das funções sociais ${ }^{11}$.

Dessa forma, os profissionais da ESF devem ampliar a rede de suporte social dos idosos viúvos. Isto pode ser feito por meio do incentivo para maximizar o relacionamento com as outras pessoas do círculo familiar e estimular a participação de atividades na comunidade que lhes tragam prazer e satisfação.
Predominou a escolaridade de 4-8 anos com menor percentual entre os idosos diabéticos com indicativo de depressão em comparação aos sem. Estudo realizado na zona urbana de Taiwan com idosos diabéticos constatou que 30,1\% não possuíam escolaridade ${ }^{20}$. Outro inquérito, desenvolvido no sul do Brasil, constatou que 64,4\% dos idosos com DM e sem depressão apresentavam menos de quatro anos de estudo ${ }^{16}$. Estes resultados divergem da presente investigação; porém, essas diferenças podem ser elucidadas pelos aspectos culturais peculiares de cada região ou país.

O baixo nível de instrução escolar de idosos pode refletir na aprendizagem quanto ao autocuidado em função da dificuldade no acesso a informações, bem como na capacidade de buscar os serviços dos quais necessitam. Somado a isso, esses indivíduos podem apresentar algumas limitações no entendimento das condutas terapêuticas ${ }^{21}$. Nesse sentido, o profissional de saúde, durante o atendimento ao idoso, deve adequar a linguagem para favorecer o seu entendimento, visando a uma comunicação efetiva.

Predominaram os que recebiam até um salário mínimo, com percentual superior entre os idosos diabéticos com indicativo de depressão em relação aos sem, conforme mostra a Tabela 1 . A prevalência da baixa renda dos idosos diabéticos com e sem indicativo de depressão deste estudo corrobora os dados demográficos do censo realizado em 2009, em que 43,2\% dos idosos brasileiros recebiam até um salário mínimo ${ }^{1}$. Sabe-se que o menor nível de renda dos idosos diabéticos pode representar um fator dificultador na adesão ao tratamento medicamentoso e no acesso à dieta adequada ${ }^{22}$.

Entre os idosos, o maior percentual aposentou-se por idade em ambos os grupos. Contudo, destacam-se $7(21,9 \%)$ idosos com indicativo de depressão que se aposentaram por problemas de saúde, de acordo com a Tabela 1. Segundo o Instituto Nacional do Seguro Social (INSS), a maioria dos idosos $(65,5 \%)$ da zona rural aposentou-se por idade ${ }^{23}$. Cabe ressaltar que essa população pode solicitar a aposentadoria com cinco anos a menos do que na zona urbana, sendo estipulados 55 anos para mulheres e 60 anos para homens ${ }^{23}$.

Parte expressiva de idosos diabéticos com indicativo de depressão referiu ter se aposentado por problemas de saúde, seguindo os sem tendências depressivas. Evidencia-se que as doenças crônicas, frequentemente encontradas nos idosos, podem gerar sintomatologia depressiva nesses indivíduos ${ }^{24}$, podendo contribuir para a deterioração do seu estado de saúde e, com isso, intervir no motivo de aposentadoria.

Os dados relacionados à saúde dos idosos diabéticos estão relacionados na Tabela 2 . 
TABELA 2: Distribuição das variáveis de saúde dos idosos diabéticos da zona rural com e sem indicativo de depressão. Uberaba, 2012. $(N=72)$

\begin{tabular}{|c|c|c|c|c|c|c|}
\hline \multirow{2}{*}{ Variáveis } & \multicolumn{2}{|c|}{ Com } & \multicolumn{2}{|c|}{ Sem } & \multirow{2}{*}{$x^{2}$} & \multirow{2}{*}{$p$} \\
\hline & $f$ & $\%$ & $f$ & $\%$ & & \\
\hline \multicolumn{7}{|l|}{ Autopercepção de saúde } \\
\hline Péssima & 2 & 6,2 & 2 & 2,8 & \multirow{5}{*}{11,08} & \multirow{5}{*}{0,03} \\
\hline Má & 5 & 15,6 & 2 & 2,8 & & \\
\hline Regular & 19 & 59,4 & 39 & 54,2 & & \\
\hline Boa & 6 & 18,8 & 24 & 33,3 & & \\
\hline Ótima & - & - & 5 & 6,9 & & \\
\hline \multicolumn{7}{|c|}{ Saúde comparada há 12 meses } \\
\hline Pior & 15 & 46,9 & 19 & 26,4 & \multirow{3}{*}{5,58} & \multirow{3}{*}{0,06} \\
\hline Igual & 8 & 25 & 34 & 47,2 & & \\
\hline Melhor & 9 & 28,1 & 19 & 26,4 & & \\
\hline \multicolumn{7}{|c|}{ Saúde em relação a outros da mesma idade } \\
\hline Pior & 6 & 18,8 & 8 & 11,1 & \multirow{4}{*}{2,8} & \multirow{4}{*}{0,42} \\
\hline Igual & 10 & 31,2 & 27 & 37,5 & & \\
\hline Melhor & 12 & 37,5 & 33 & 45,8 & & \\
\hline Ignorado & 4 & 12,5 & 4 & 5,6 & & \\
\hline \multicolumn{7}{|l|}{ Consulta mensal } \\
\hline Sim & 15 & 46,9 & 34 & 47,2 & \multirow{2}{*}{0,01} & \multirow{2}{*}{0,97} \\
\hline Não & 17 & 53,1 & 38 & 52,8 & & \\
\hline \multicolumn{7}{|l|}{ Tempo de diagnóstico $(*)$} \\
\hline$\leq 5$ & 8 & 26,7 & 29 & 42,6 & \multirow{4}{*}{3,18} & \multirow{4}{*}{0,36} \\
\hline $06-10$ & 7 & 23,3 & 14 & 20,6 & & \\
\hline $11-20$ & 11 & 36,7 & 15 & 22 & & \\
\hline$>20$ & 4 & 23,3 & 10 & 14,7 & & \\
\hline \multicolumn{7}{|l|}{ Complicações(**) } \\
\hline Sim & 16 & 51,6 & 18 & 25,7 & \multirow{2}{*}{12,52} & \multirow{2}{*}{0,03} \\
\hline Não & 15 & 48,4 & 52 & 74,3 & & \\
\hline \multicolumn{7}{|l|}{ Uso de medicamentos } \\
\hline Sim & 28 & 87,5 & 70 & 97,2 & \multirow{2}{*}{3,49} & \multirow{2}{*}{0,06} \\
\hline Não & 4 & 12,5 & 2 & 2,8 & & \\
\hline \multicolumn{7}{|l|}{ Tipo de medicação } \\
\hline Insulina & 3 & 9,4 & 2 & 2,8 & \multirow{4}{*}{8,07} & \\
\hline Hipoglicemiante oral & 20 & 62,5 & 63 & 87,5 & & \\
\hline Combinado & 5 & 15,6 & 4 & 5,5 & & 0,04 \\
\hline Nenhum & 4 & 12,5 & 3 & 4,2 & & \\
\hline
\end{tabular}

$(*)$ Não responderam 2 idosos com indicativo de depressão e 4 sem indicativo.

(**) Não responderam 1 idoso com indicativo de depressão e 2 sem indicativo.

Observou-se maior proporção de idosos diabéticos com indicativo de depressão com autopercepção de saúde negativa em comparação aos sem indicativo $\left(X^{2}=11,08 ; p=0,03\right)$, conforme mostra a Tabela 2 . Os resultados obtidos apresentaram incongruência com investigação conduzida na zona urbana em um município de Minas Gerais, com idosos diabéticos, a qual verificou que $50 \%$ daqueles com indicativo de depressão e 83,5\% sem indicativo referiram como bom seu estado de saúde ${ }^{17}$. Essa divergência pode ser justificada pelas diferenças de área de residência dos idosos.

Evidencia-se que a autopercepção desfavorável da condição de saúde de indivíduos diabéticos ocorre devido às limitações e complicações decorrentes da doença $a^{25}$. Somadas ao indicativo de depressão, poderiam justificar a maior proporção de idosos que considerou seu estado de saúde ruim.

Apesar de os idosos diabéticos com indicativo de depressão considerem seu estado de saúde pior do que há 12 meses e aqueles sem indicativo considerarem-na igual, não houve diferença significativa entre os grupos $\left(X^{2}=5,58 ; p=0,06\right)$, segundo a Tabela 2 . A pior autopercepção de saúde dos idosos diabéticos com indicativo de depressão pode decorrer da falta de intervenção no início dos sintomas, ocasionando em um agravamento do quadro ao decorrer dos dias e, consequentemente, em piora do estado de saúde. Assim, pontua-se a necessidade da realização do diagnóstico da depressão precocemente, para que seja oferecido o tratamento adequado. 
Quando compararam sua saúde com a de outras pessoas, o maior percentual incidiu sobre os idosos diabéticos de ambos os grupos que relatam estar melhor, sem diferença significativa entre eles $\left(X^{2}=1,49 ; p=0,47\right)$, de acordo com a Tabela 2. Destaca-se que os indivíduos com depressão tendem a apresentar baixa autoestima, contribuindo em pior autopercepção do estado de saúde ${ }^{17}$, comparado a outras pessoas da mesma idade.

A maioria dos idosos diabéticos com e sem indicativo de depressão não compareciam a consultas mensais para controle da saúde, sem diferença entre os grupos $\left(X^{2}=0,01 ; p=0,97\right)$, conforme mostra a Tabela 2. Destaca-se que 25(80,6\%) idosos com indicativo de depressão e $47(65,3 \%)$ sem tal tendência possuíam cadastro no Hiperdia. Contudo, predominaram as consultas particulares em ambos os grupos, com $10(31,2 \%)$ no $1^{\circ}$ grupo e $22(30,6 \%)$ no $2^{\circ}$ grupo (sem tendências depressivas). A partir destes dados, destaca-se a importância dos profissionais da ESF realizarem visitas domiciliares, possibilitando a busca ativa desses idosos e, assim, o seu acompanhamento. Contudo, a falta de transporte para os profissionais realizarem as visitas, inadequação da infraestrutura das UBS, escassez de materiais necessários para a realização do trabalho, além da falta de entendimento por parte da população em relação ao funcionamento da política da $\mathrm{ESF}^{26}$ podem dificultar o acompanhamento destes idosos.

A despeito de não haver diferença entre os grupos $\left(X^{2}=3,18 ; p=0,36\right)$, a maioria dos idosos diabéticos com indicativo de depressão possuía maior tempo de diagnóstico de DM (11-20 anos) em relação aos sem indicativo (0-5 anos), conforme a Tabela 2 . Entre os idosos diabéticos, evidencia-se uma dificuldade de aceitar o diagnóstico, exigindo, sobretudo, uma restrição e modificação de hábitos, imposto pelo tratamento. Por isso, os indivíduos com DM tendem a alternar entre momentos de impotência/desânimo e momentos de confiança no tratamento ${ }^{7}$. Assim, faz-se necessário que os profissionais, por meio da educação em saúde, discutam com os idosos diabéticos sobre as dificuldades vivenciadas no cotidiano, bem como modos de enfrentamento da doença, minimizando o desenvolvimento de sentimentos negativos em torno da cronicidade da doença e do tratamento adequado. $O$ atendimento deve valorizar a prevenção com vistas ao envelhecimento saudável e à qualidade de vida ${ }^{27}$. Nesse contexto, ressalta-se que intervenções educativas de enfermagem podem auxiliar na melhoria do estado de saúde e parâmetros clínicos de indivíduos com doenças crônicas ${ }^{28}$.

Houve maior proporção de idosos diabéticos com indicativo de depressão com complicações em comparação aos sem esse indicativo $\left(X^{2}=12,52 ; p=0,03\right)$, de acordo com a Tabela 2. Evidencia-se que a gravidade e o número de complicações do DM podem estar relacionados ao aumento dos sintomas depressivos ${ }^{12}$. Nesse sentido, deve-se realizar acompanhamento periódico dos idosos diabéticos, buscando identificar a presença de complicações.

As complicações prevalentes entre idosos diabéticos com indicativo de depressão foram os problemas de visão - 15(55,6\%) e cardíacos - 7(25,9\%), enquanto naqueles sem indicativo foram os problemas de visão $52(81,2 \%)$ e renal - 6(9,4\%). Esses achados divergem de estudo realizado no sul do Brasil, no qual observou-se que as comorbidades prevalentes em idosos diabéticos com depressão foram problemas vasculares $(83,7 \%)$, musculares $(81,3 \%)$ e renais $(54,4 \%)$; naqueles sem depressão, houve maior percentual de complicações musculares $(57,7 \%)$, vasculares $(42,2 \%)$ e renais $(26,8 \%)^{16}$.

$\mathrm{O}$ uso de medicamentos para diabetes não se diferenciou entre os grupos $\left(X^{2}=3,49 ; p=0,06\right)$, segundo a Tabela 2 .

Resultado oposto foi encontrado em estudo realizado na zona rural da Carolina do Norte, Estados Unidos, o qual verificou que $51 \%$ dos idosos diabéticos com indicativo de depressão e 67,3\% daqueles sem indicativo não utilizavam medicações ${ }^{18}$. Destaca-se a adoção racional da terapia farmacológica, visando evitar uso desnecessário, efeitos colaterais e interações dos componentes medicamentosos. Assim, faz-se necessário que a equipe de saúde utilize meios para o tratamento e prevenção com práticas saudáveis ${ }^{8,11}$.

Houve maior proporção de idosos diabéticos com indicativo de depressão utilizando terapia combinada e insulina se comparada aos sem indicativo $\left(\mathrm{X}^{2}=8,07\right.$; $p=0,04)$, conforme mostra a Tabela 2 . Um inquérito realizado na zona rural nos Estados Unidos com idosos diabéticos apresentou resultados opostos: verificou-se que aqueles pacientes com depressão apresentaram percentual inferior no uso da terapia combinada $(25,5 \%)$ em relação àqueles que não tinham depressão $(28,0 \%)$; porém não houve diferença significativa entre esses grupos ${ }^{18}$.

No entanto, a maior proporção de indicativo de depressão entre idosos diabéticos em uso de terapia combinada do presente estudo pode estar relacionada ao aumento do número de medicamentos que, muitas vezes, são usados em diferentes horários do dia e ao desconforto causado pela administração da insulina.

A distribuição das variáveis de lazer da população estudada está relacionada na Tabela 3.

Predominaram, em ambos os grupos, os idosos que realizavam atividades de lazer, sem diferença significativa $\left(\mathrm{X}^{2}=3,49 ; p=0,06\right)$ entre eles conforme a Tabela 3. Entretanto, menor percentual de idosos diabéticos com indicativo de depressão referiu realizar dança, atividades manuais, jogos, leitura e assistir televisão em relação aos sem indicativo, segundo a Tabela 3. Sabe-se que essas atividades 
TABELA 3: Distribuição das variáveis de lazer dos idosos diabéticos com e sem indicativo de depressão, residentes na zona rural. Uberaba, 2012. ( $N=72)$

\begin{tabular}{|c|c|c|c|c|c|c|}
\hline \multirow{2}{*}{ Variáveis } & \multicolumn{2}{|c|}{ Com } & \multicolumn{2}{|c|}{ Sem } & \multirow{2}{*}{$x^{2}$} & \multirow[b]{2}{*}{$p$} \\
\hline & $f$ & $\%$ & $f$ & $\%$ & & \\
\hline \multicolumn{7}{|l|}{ Lazer } \\
\hline Sim & 28 & 87,5 & 70 & 97,2 & \multirow{2}{*}{3,49} & \multirow{2}{*}{0,06} \\
\hline Não & 4 & 12,5 & 2 & 2,8 & & \\
\hline \multicolumn{7}{|l|}{ Dançar(*) } \\
\hline Sim & 3 & 9,7 & 17 & 23,6 & \multirow{2}{*}{2,69} & \multirow{2}{*}{0,1} \\
\hline Não & 28 & 90,3 & 55 & 76,4 & & \\
\hline \multicolumn{7}{|c|}{ Atividade manual $(*)$} \\
\hline Sim & 10 & 32,3 & 32 & 44,4 & \multirow{2}{*}{1,33} & \multirow{2}{*}{0,25} \\
\hline Não & 21 & 67,7 & 40 & 55,6 & & \\
\hline \multicolumn{7}{|l|}{$\operatorname{Jogos}(*)$} \\
\hline Sim & 1 & 3,2 & 11 & 15,3 & \multirow{2}{*}{3,74} & \multirow{2}{*}{0,05} \\
\hline Não & 30 & 96,8 & 61 & 84,7 & & \\
\hline \multicolumn{7}{|l|}{$\operatorname{Ler}(*)$} \\
\hline Sim & 8 & 25,8 & 27 & 37,5 & \multirow{2}{*}{1,32} & \multirow{2}{*}{0,25} \\
\hline Não & 23 & 74,2 & 45 & 62,5 & & \\
\hline \multicolumn{7}{|l|}{ Rádio } \\
\hline Sim & 13 & 40,6 & 48 & 66,7 & \multirow{2}{*}{6,19} & \multirow{2}{*}{0,01} \\
\hline Não & 19 & 59,4 & 24 & 33,3 & & \\
\hline \multicolumn{7}{|l|}{ Televisão } \\
\hline Sim & 20 & 62,5 & 56 & 77,8 & \multirow{2}{*}{2,63} & \multirow{2}{*}{0,1} \\
\hline Não & 12 & 37,5 & 16 & 22,2 & & \\
\hline
\end{tabular}

(*) 1 idoso com indicativo não respondeu.

podem proporcionar interação social, o que contribui para o desenvolvimento de sua própria autonomia e controle ou diminuição dos sintomas depressivos, angústia e estresse ${ }^{29}$. Além disso, o idoso deve se envolver em atividades que o façam sentir-se útil, tragam prazer e felicidade ${ }^{19}$.

Houve maior proporção de idosos diabéticos sem indicativo de depressão que ouvia rádio comparado aos com indicativo $\left(\mathrm{X}^{2}=2,63 ; p=0,01\right)$, conforme mostra a Tabela 3. Este fato pode estar relacionado à falta de interesse ou prazer pelas atividades que antes eram de costume, sendo este um dos sintomas comuns da depressão $0^{30}$. Salienta-se que, em ambos os grupos, $23(74,2 \%)$ com e $56(77,8 \%)$ sem indicativo de depressão, estavam satisfeitos com suas atividades de lazer. O lazer é essencial para manter o idoso inserido na sociedade; desse modo, esse aspecto deve ser verificado pela equipe de saúde, em seu processo de trabalho. Deve-se identificar quais atividades os idosos têm interesse e buscar estratégias conjuntas (cliente/ profissional) visando sua implementação.

\section{CONClusão}

Em sua maioria, os idosos diabéticos eram do sexo feminino, na faixa etária entre 60-70 anos, moravam com o esposo ou companheiro, tinham 4-8 anos de estudo, renda de até um salário mínimo; destacaram-se os sujeitos sem indicativo de depressão por terem se aposentado por idade.

Os idosos diabéticos com indicativo de depressão apresentaram, proporcionalmente, pior autopercepção da saúde, mais complicações relacionadas ao DM e maior uso de medicamentos combinados e insulina comparados aos sem indicativo de depressão. Em relação às atividades de lazer, menor proporção de idosos com tendências depressivas ouviam rádio em comparação àqueles sem tais tendências.

Destacam-se como limitações deste estudo as morbidades autorreferidas (sem documentos comprobatórios) e o delineamento transversal que não permite estabelecer relação de causa e efeito entre as variáveis investigadas. Ainda assim, foi possível aprofundar o conhecimento sobre as características sociodemográficas, bem como o perfil de saúde dos idosos com DM residentes na zona rural, com e sem indicativo de depressão.

Contudo, os resultados desta pesquisa denotam a necessidade de acompanhamento dos idosos com indicativo de depressão visando estabelecimento de diagnóstico. A partir daí, poderá ser planejada intervenção direcionada às peculiaridades de cada idoso. Deve-se priorizar ações que visem identificar o impacto da doença sobre o cotidiano bem como buscar estratégias para ampliação das opções de lazer, de acordo com a preferência dos idosos. 


\section{REFERÊNCIAS}

1.Instituto Brasileiro de Geografia e Estatística (IBGE). Uma análise das condições de vida da população brasileira 2010. Rio de Janeiro: IBGE; 2010. [citado em 27 jan 2015]. Disponível em: http://www.ibge.gov.br/home/estatistica/ populacao/condicaodevida/indicadoresminimos/sinteseindicsociais2010/SIS_2010.pdf.

2.Ministério da Saúde $(\mathrm{Br})$. Atenção à saúde da pessoa idosa e envelhecimento. Série Pactos pela Saúde. Brasília (DF): Ministério da Saúde; 2010.

3.Martins CR, Albuquerque FJB, Gouveia CNNA, Rodrigues CFF, Neves MTS. Avaliação da qualidade de vida subjetiva dos idosos: uma comparação entre os residentes em cidades rurais e urbanas. Estud interdiscip envelhec. 2007; 11:135-54.

4.Sjolund BM, Nordberg G, Wimo A, Strauss EV. Morbidity and physical functioning in old age: differences according to living area. J Am Geriatr Soc. 2010; 58:1855-62.

5.Viegas-Pereira APF, Rodrigues RN, Machado CJ. Fatores associados à prevalência de diabetes auto-referido entre idosos de Minas Gerais. Rev Bras Estud Popul. 2008; 25:365-76.

6.American Diabetes Association (ADA). Diagnosis and classification of Diabetes Mellitus. Diabetes Care. 2008; 31:55-60.

7.Peres DS, Santos MA, Zanetti ML, Ferronato AA. Dificuldades dos pacientes diabéticos para o controle da doença: sentimentos e comportamentos. Rev Latino-Am Enfermagem. 2007; 15:1105-12.

8.Nascimento AB, Chaves EC, Grossi SAA, Lottenberg SA. A relação entre polifarmácia, complicações crônicas e depressão em portadores de diabetes mellitus tipo 2. Rev esc enferm USP. 2010; 44:40-6.

9.Rajkumar AP, Thangadurai P, Senthilkumar P, Gayathri $\mathrm{K}$, Prince M, Jacob KS. Nature, prevalence and factors associated with depression among the elderly in a rural south Indian community. Int Psychogeriatr. 2009; 21:372-8.

10.O'Connor PJ, Crain AL, Rush WA, Hanson AM, Fischer LR, Kluznik JC. Does diabetes double the risk of depression?. Ann Fam Med. 2009; 7:328-35.

11.Fernandes MGM, Nascimento NFS, Costa KNFM. Prevalência e determinantes de sintomas depressivos em idosos atendidos na atenção primária de saúde. Rev RENE. 2010; 11:19-27.

12.Moreira RO, Amâncio APRL, Brum HR, Vasconcelos DL, Nascimento GF. Sintomas depressivos e qualidade de vida em pacientes diabéticos tipo $2 \mathrm{com}$ polineuropatia distal diabética. Arq Bras Endocrinol Metab. 2009; 53:1103-11.

13.Bertolucci PHF, Brucki SMD, Campacci SR, Juliano Y. O mini-exame do estado mental em uma população geral: impacto da escolaridade. Arq Neuro-Psiquiatria. 1994; 52:1-7.

14.Frank MH, Rodrigues NL. Depressão, ansiedade, outros distúrbios afetivos e suicídio. In: Freitas EV, Py L. Tratado de geriatria e gerontologia. $2^{\mathrm{a}}$ ed. Rio de Janeiro: Guanabara Koogan; 2006. p. 376-87.

15.Ramos L R. Growing old in São Paulo, Brazil: assess- ment of health status and family support of the elderly of different socio-economic strata living in the community [tese de doutorado]. London: London School of Hygiene and Tropical Medicine, University of London, 1987.

16.Blay SL, Fillenbaum GG, Marinho V, Andreoli SB, Gastal FL. Increased health burden associated with comorbid depression in older Brazilians with diabetes. J Affect Disord. 2011; 134: 77-84.

17.Faria ACNB, Barreto SM, Passos VMA. Sintomatologia depressiva em idosos de um plano de saúde. Rev Med Minas Gerais. 2008; 18:175-82.

18.Bell RA, Andrews JS, Arcury TA, Snively BM, Golden SL, Quandt SA. Depressive symptoms and diabetes self-management among rural older adults. Am J Health Behav. 2010; 34:36-44.

19.Tavares DMS, Gávea Junior SA, Dias FA, Santos NMF, Oliveira PB. Qualidade de vida e capacidade funcional de idosos residentes na zona rural. Rev RENE. 2011; 12:895-903.

20.Bai YL, Chiou CP, Chang YY, Lam HC. Correlates of depression in type 2 diabetic elderly patients: a correlational study. International Journal of Nursing Studies. 2008; 45:571-9.

21.Grillo MFF, Gorini MIPC. Caracterização de pessoas com Diabetes Mellitus tipo 2. Rev Bras Enferm. 2007; 60:49-54.

22.Tavares DMS, Rodrigues FR, Silva CGC, Miranzi SSC. Caracterização de idosos diabéticos atendidos na atenção secundária. Ciênc saúde coletiva. 2007; 12:1341-52.

23. Ministério da Previdência Social (Br). Anuário Estatístico da Previdência Social. 2010. [citado em 27 jan 2015]. Disponível em: http://www.previdencia.gov.br/arquivos/ office/3_111202-105619-646.pdf.

24.Borges DT, Dalmolin BM. Depressão em idosos de uma comunidade assistida pela estratégia de saúde da família em Passo Fundo, RS. Rev Bras Med Fam Comunidade. 2012; 7 (23):75-82.

25.Francisco PMSB, Belon AP, Barros MBA, Carandina L, Alves MCGP, Goldbaum M, Cesar CLG. Diabetes auto-referido em idosos: prevalência, fatores associados e práticas de controle. Cad Saúde Pública. 2010; 26:175-84. 26.Silva SA, Oliveira F, Spinola CM, Poleto VC. Atividades desenvolvidas por enfermeiros no PSF e dificuldades em romper o modelo flexneriano. Rev Enferm Centro $\mathrm{O}$ Min. 2011; 1:30-9.

27. Oliveira MAS, Menezes TMO. A enfermeira no cuidado ao idoso na estratégia saúde da família: sentidos do vivido. Rev enferm UERJ. 2014; 22:513-8.

28.Luna NSA, Baeza MR, Castell EC, Santos FC, David HL, Castilho MMA. Intervención educativa: implementación de la agencia de autocuidado y adherencia terapêutica desde la perspectiva del paciente diabético. Rev enferm UERJ. 2013; 21:289-94.

29.Navarro FM, Rabelo JF, Faria ST, Lopes MCL, Marcon SS. Percepção de idosos sobre a prática e a importância da atividade física em suas vidas. Rev Gaúcha Enferm. 2008; 29:596-603.

30.Carreira L, Botelho MR, Matos PCB, Torres MM, Salci M. Prevalência de depressão em idosos institucionalizados. Rev enferm UERJ. 2011; 19:268-73. 